
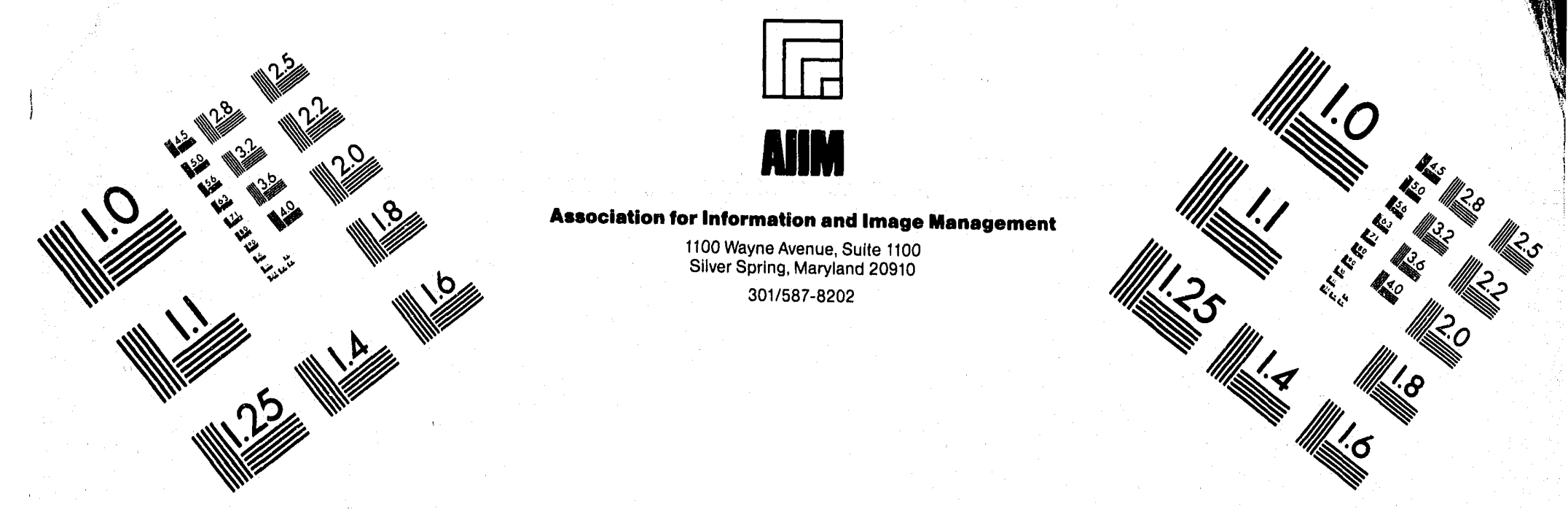

\title{
Centimeter
}

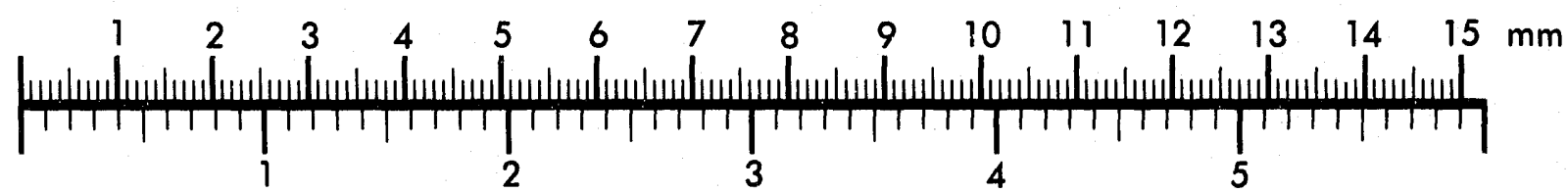
Inches
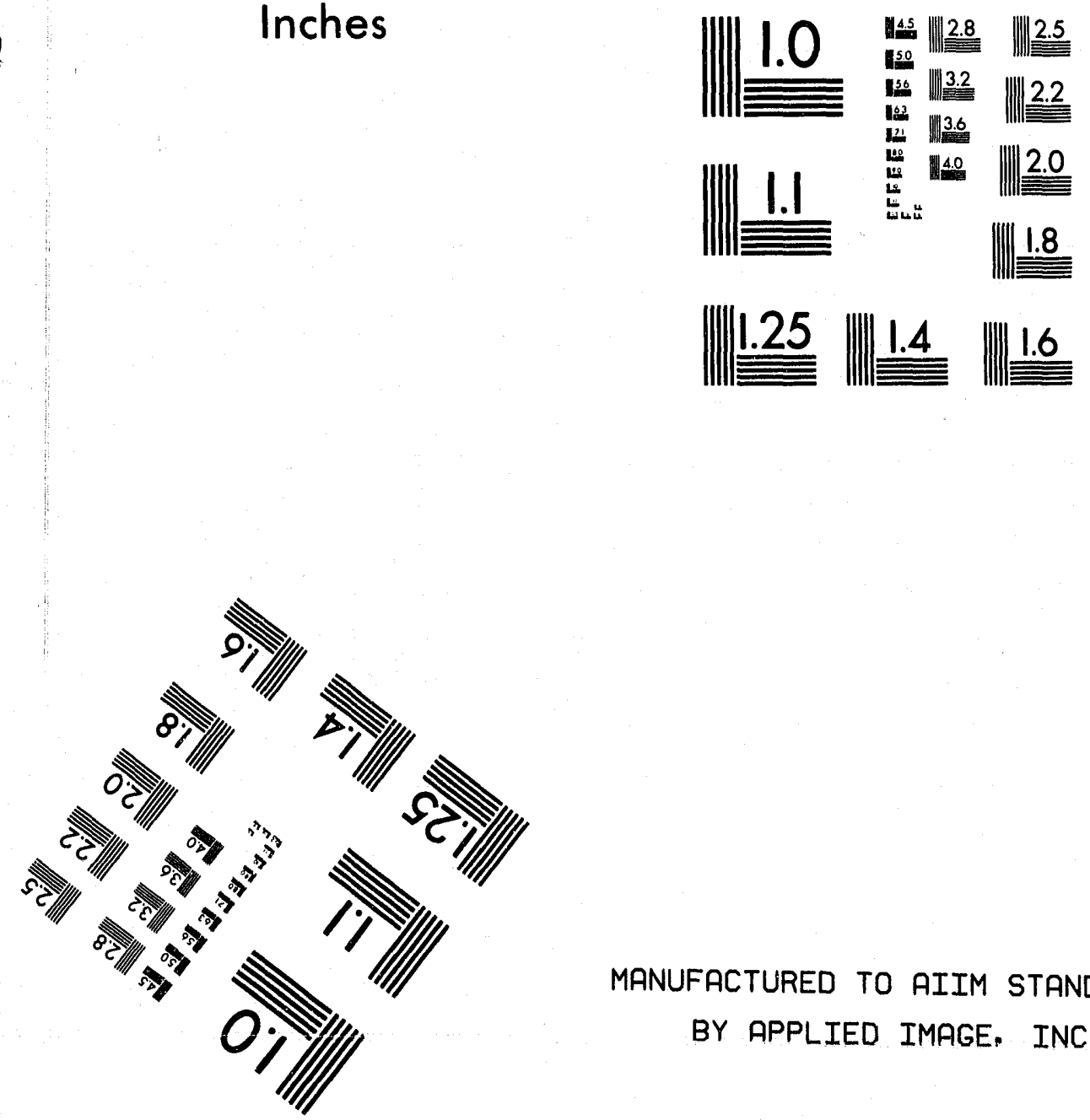

MANUFACTURED TO AIIM STANDARDS

BY APPLIED IMAGE, INC.

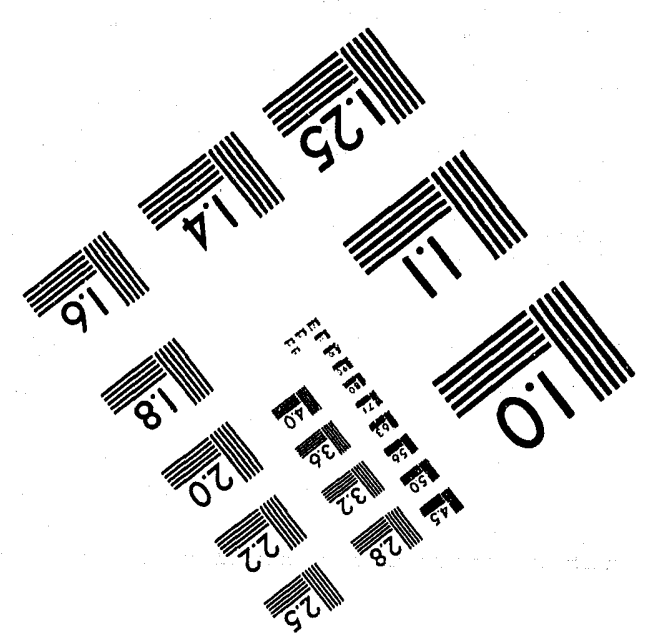



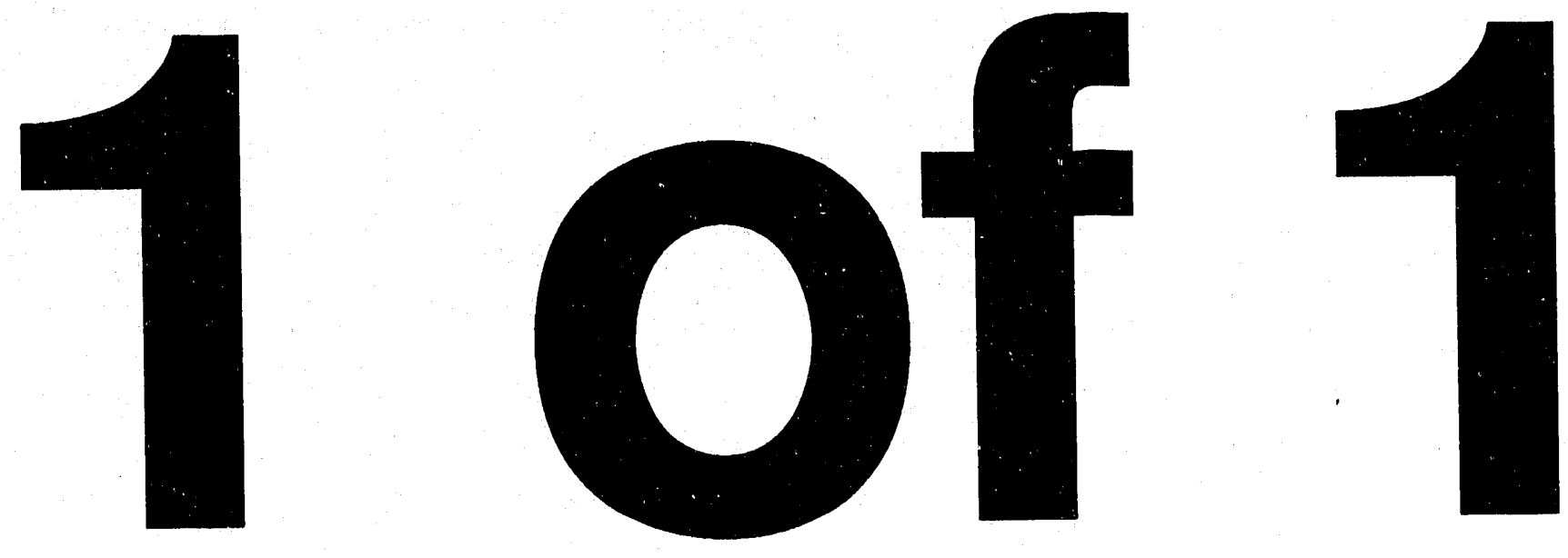
LBL-32612

UC-414

\title{
Scintillating Tile Fabrication for the 4x4 Calorimeter Test Station Module
}

\author{
Matthew D. Hoff \\ Lawrence Berkeley Laboratory \\ University of California \\ Berkeley, Ca. 94720 \\ June 23, 1992 \\ SDC 92-288 \\ LBL-SDC-CAL 92-21 \\ WBS 2.2 \\ Category Code 2.02.09 \\ M7287
}

This work was supported by the Director, Office of Energy Research, Office of High Energy Nuclear Physics, Division of High Energy Physics, Office of the Superconducting Super Collider of the U.S. Department of Energy, under Contract No. DE-ACO3-76SF00098

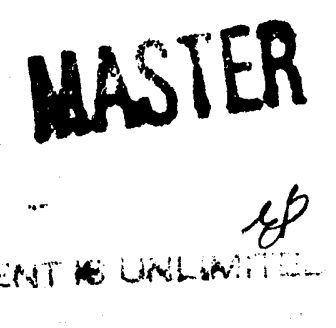




\section{Scintillating Tile Fabrication for the 4x4 Calorimeter Test Station Module}

LBL has proposed a new conceptual design for the SDC Endcap

Electromagnetic Calorimeter. To obtain the resolution needed, the design is "monolithic", with no structural material nor mechanical joints between neighboring scintillator towers. To explore its actual physics performance, a representative, full depth, 16 tower portion of the ECEM was built for beamline testing. This " $4 \times 4$ Test Station Module" also provided valuable experience in the machining and assembly of scintillating tiles. The following is a record of the tile fabrication techniques used, and the lessons learned.

The 4 millimeter thick scintillating tile material arrived from Japan in sheets $50 \mathrm{~cm}$ square, packed in a wooden crate. The crate was taken to Building 77 (Main Shop) where all the machining steps of the tile fabrication took place between April 6 and April 30,1992. The protective paper face sheets were left in place throughout the machining operations.

The tiles were first bandsaw cut $1 / 8$ inch oversized ( $1 / 16$ per side) using a new 4 T.P.I. skip tooth blade run at 2000 feet per minute. Three, $50 \mathrm{~cm}$ square sheets were stacked and cut at a time. Each sheet was cut into 16 tiles. Previous to cutting, the band saw was cleaned to remove grease and chips, and cardboard was taped to the table to prevent scratching. 25 sheets were cut up requiring an estimated 3 hours of work.

Next, the rough tiles were set up in a milling machine to be squared and cut to their finished size. Loose protective paper and chips were removed before a stack of 11 tiles were placed against a pair of 4 inch angle plates and trimmed. A 3 inch fly cutter with a carbide blade spinning at 2000 R.P.M. traveling at 15 inches per minute did the cutting. Each cut removed .030 inch of material or less. The stack was rotated after each pass. (The machinist was afraid that a cut of more than .030 would cause chipping.) Each stack took about one hour to machine, 34 stacks, for a total of 35 hours. A random inspection of five tiles after machining indicated a tolerance of $+.002 /-.004$ on the overall size was attained.

The next step was to cut the groove for the wave shifting fiber. A vacuum chuck was fabricated using an aluminum block to hold the tiles flat during machining. Using a numerically controlled milling machine, a .031 diameter 
carbide two flute end mill with a .025 radius on the end made three passes in the "scientifically determined" groove pattern. A precision high speed head spinning at 9000 R.P.M. with a feed rate of 15 inches per minute cut its first pass .030 deep. The second pass at .060 deep and the third pass at .065 deep. A special manifold blowing filtered compressed air was fabricated to cool the end mill and clear the chips. This step of the fabrication required about 40 hours total, or about 6.4 minutes each tile. After all the tiles were end milled, the milling machine was retooled with a two flute .051 diameter carbide ball mill cutter with a .028 inch diameter shank. One pass was made with the ball mill cutter at 9000 R.P.M. traveling 15 inches per minute at a depth of .065 inches. At the end of the pass the ball mill was pulled straight out of the tile, instead of retracing the groove, to save time. The same air manifold was used for cooling during this step. The ball mill operation required about 20 hours to run or about 3.2 minutes per tile.

End and ball mill cutter life was difficult to ascertain. Five .031 end mills were used. Three end mills broke immediately after entering the tile, one broke after a few tiles and the last mill ran till the end, grooving hundreds of tiles. Two .051 ball mills were used. The first ball mill lasted about 200 tiles until the machinist "crashed" the mill into the fixture. The second ball mill lasted through the end of the run and showed no sign of wear.

The final machining process was the drilling of the "spoke hole". The vacuum chuck was refitted with hold down clamps. This method works best when drilling holes through the tile. The precision high speed head was removed and a .125 diameter end mill ground to a drill point was spun at 1000 R.P.M. to make the hole. The drill was advanced at 2.5 inches per minute with two backout steps to "peck" the hole through the tile. Hole drilling took about 12 hours. The tiles required 22 different hole locations with about 16 tiles receiving each individual hole location. A total of about 360 tiles survived to the end of the hole drilling operation. About 20 tiles were set aside during fabrication because of minor scratches and chips.

Through out the entire fabrication operation the tiles were handled with gloves and protected from scratches, metal chips, oils, and cutting fluids. The lead machinist on the job was Bob Shannon, with support from Dave Robinson, Manuel Pereira and Dent Trail. Dave Fraser, the tool grinder, custom made the .051 ball mill cutters. Jack Smith was the shop supervisor, and Chip Hollister lent technical support.

After fabrication, the tiles were moved to the Assembly Shop and given to Roy Oki to paint. Paint was applied only around the exposed $4 \mathrm{~mm}$ edge using a cotton swab with a piece of open cell foam tied over the cotton tip. All the tiles were painted individually, requiring about 3 minutes each. Using the special Bicron $\mathrm{BC}-620$ reflective coating, each tile was initially covered with two coats of paint. The paint added an average of .003 inch to each side of the 
tile. After further inspection it was deemed necessary to add a third coat of paint. The third layer of paint added another .001 inch per side bringing the total paint thickness to .004 inch per side, adding .008 inch total. The tiles required about 24 hours to cover with the three layers of paint.

The next step after painting was to peel off the protective paper. This step became much more problematic and time consuming than first imagined. The first problem occurred when peeling off the protective paper. The excess paint that ran over onto the protective paper flaked into small pieces and was then staticly attracted to the face of the tile. (The static charge on the tile was created by peeling off the paper.) To remove the paint chips, a small paint brush was employed. The brush only succeeded in removing the big flakes and pushing the small flakes around the surface. The paint brush also left light scratches on the tiles. To alleviate the previous problems, Heather Kelly of the Assembly Shop was put to work scraping the extra paint off the protective paper with a single sided razor blade before removing the paper. This step required about seven hours and the paint chips were blown off with dry nitrogen. Next, the protective paper was slowly peeled away under a steady flow of bottled dry nitrogen ( 30 P.S.I. ) with an ionizing nozzle. This step of the operation required about 20 hours. The tiles left this stage of the fabrication scratch free and with only a few specks of paint still clinging.

The next step in the fabrication was to wrap the tiles in $.0005^{\prime \prime}$ thick aluminized mylar. The aluminized mylar was cut to a rough size and clamped between two sheets of lucite, then milled to the exact size. The naked tiles were first blown off with dry nitrogen, leaving a slight static charge. The mylar would then cling to the tile as it was wrapped around four sides. A small prepunched hole in the mylar was lined up with the fiber entrance hole in the tile to allow insertion of the fiber at a later time. Once the mylar was satisfactorily aligned, regular transparent scotch tape was applied to fasten the ends where they overlapped. Three small pieces of tape were used to join mylar to mylar. The scotch tape never touched the tile. After applying the tape, the tile was labeled with its corresponding "layer letter" by writing with a soft pencil on the tape. Next the spoke hole was located and the mylar was pierced with a scalpel. Each tile required about 2.5 minutes of work or about 15 hours total.

Next a spare wave shifting fiber was inserted into the groove to insure a clear, smooth passageway for the production fiber cable. This step became a major headache. On a few tiles, the fiber slid in completely on the first try. In most cases, it required a number of tries with extra effort involving smaller diameter fibers. In the case of tile layer " $\mathrm{A}$ ", the layer that did not have the paint scrapped off prior to the removal of the protective layer, inserting the fiber was almost impossible. Our best guess as to why the fibers were so stubborn is that the excess, very fine, paint chips generated during protective paper removal deposited in the fiber groove, causing very high friction. 
About five "A" tile grooves were scraped out by hand using the .051 "diameter ball cutter. After a lot of struggle, all tiles (all layers) did accept a fiber their full length. A wild guess as to the time spent inserting fibers would be 32 hours. The wide fluctuation of time required per tile to insert the fiber, and the high frustration level, prevented us from keeping an accurate count of time spent on this step. There seemed to be many reasons for the problems encountered with putting the fibers into the tiles. We feel some of the factors are : surface finish of the groove, dirt in the groove, the shape of the fiber tip, and the diameter of the groove. We felt that a lubricant would have helped a lot, but we were unsure of a suitable candidate. We think eliminating the fourth turn in the groove pattern should be looked at next time. Most of the fibers made it to the start of the fourth turn before getting stuck. All the tile wrapping and subsequent assembly work was done in Building 50 room 2177 from May 1 thru June 1. Most of the tile wrapping was performed by Heather Kelly, with help from Matt Hoff, Mervin Wong, William Thur, and David Bintinger. Again, all tile handling was done wearing protective gloves.

Once the tile was wrapped and the groove deemed useable, Richard Kadel inserted the production fiber optic cable. This step required about 30 seconds per tile. When a layer of 16 tiles was complete, Chris Hearty individually tested each tile for light output, and ranked them from best to worst. Testing took 30 seconds per tile. The ranking was penciled onto the scotch tape of each tile therefore making it possible to place the best and worst tiles into designated towers, reserving "average" tiles for the center towers. All the tiles were loaded into the $4 \times 4$ by Matt Hoff, and fit together properly.

This work was supported by the Director, Office of Energy Research, Office of High Energy Nuclear Physics, Division of High Energy Physics, Office of the Superconducting Super Collider of the U.S. Department of Energy, under Contract No. DE-ACO3-76SF00098 

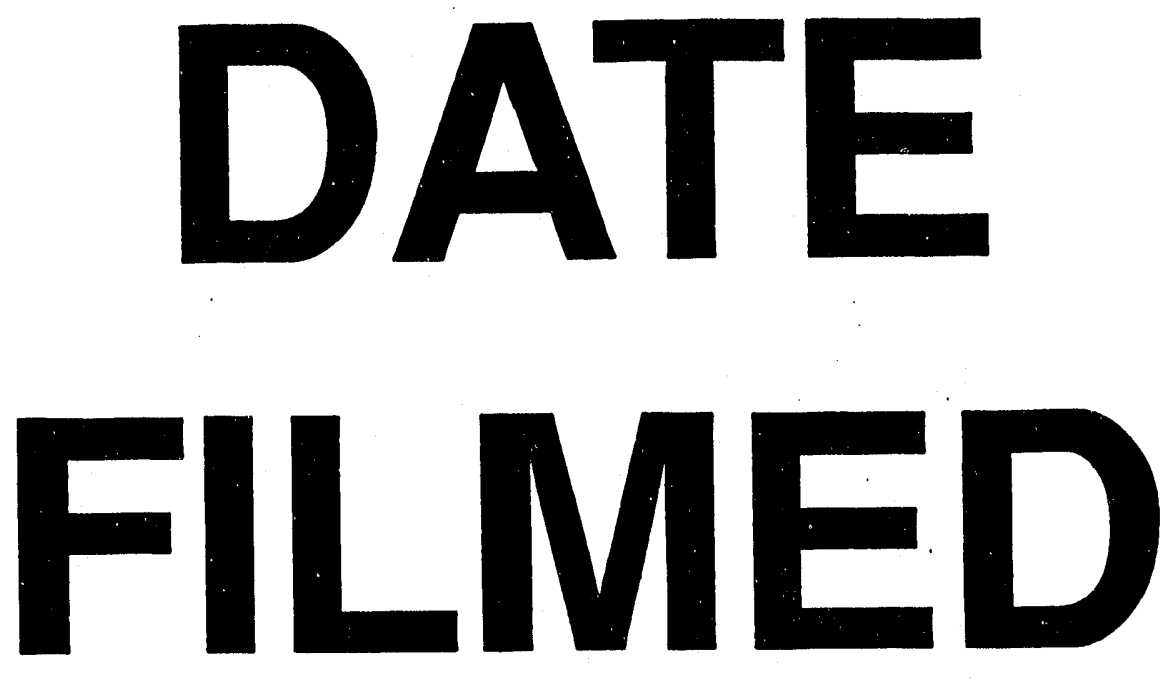

$8 / 17 / 93$
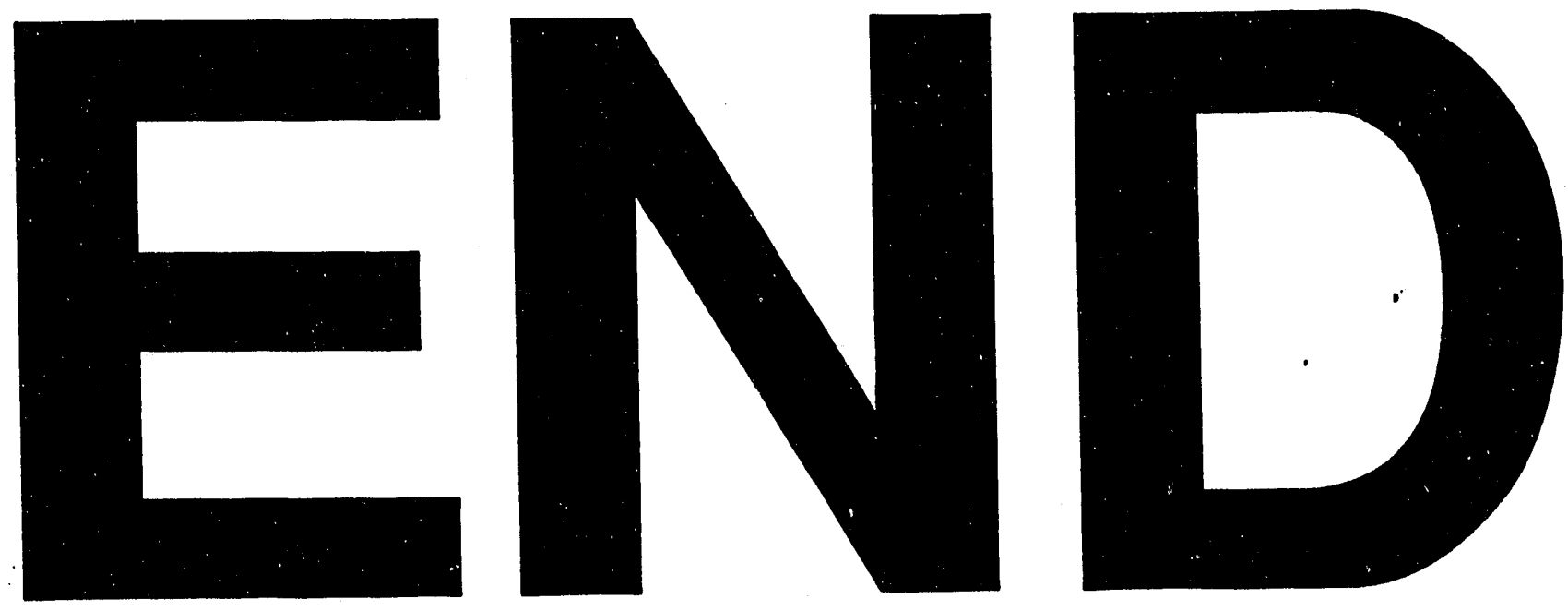
